\title{
Molecular profile of organ culture-stored corneal epithelium: LGR5 is a potential new phenotypic marker of residual human corneal limbal epithelial stem cells
}

\author{
JOANNA BRZESZCZYNSKA ${ }^{1}$, KANNA RAMAESH $^{3}$, BAL DHILLON $^{2}$ and JAMES A. ROSS ${ }^{1}$ \\ ${ }^{1}$ Tissue Injury and Repair Group, University of Edinburgh - MRC Centre for Regenerative Medicine; ${ }^{2}$ Department \\ of Clinical and Surgical Sciences, Ophthalmology Section, Princess Alexandra Eye Pavilion, Royal Infirmary of \\ Edinburgh, Edinburgh; ${ }^{3}$ Tennent Institute of Ophthalmology, Gartnavel General Hospital, Glasgow, Scotland, UK
}

Received December 15, 2011; Accepted January 16, 2012

DOI: 10.3892/ijmm.2012.904

\begin{abstract}
Long-term preservation of corneal limbal epithelium may decrease its quality and change the molecular signature of the limbal epithelial stem cells. In this study we have investigated the molecular profile of isolated corneal epithelial cells that have been in storage for an extended time. Isolated cells were characterised by the expression profile of different cytokeratins and markers of squamous metaplasia (vimentin and $\alpha$-actin). Furthermore, we examined global markers of adult stem cells including p63 $\alpha$ and ABCG 2 but also LGR5 as a novel stem cell marker. Immunocytochemical staining and PCR analysis of p63 $\alpha$, ABCG 2 and LGR5 revealed the existence of side-population cells with a stem-cell phenotype and maintenance of corneal limbal stem cell properties. LGR5 expression can be related to cellular stemness and can be considered as a new phenotypic marker of residual human corneal limbal stem cells. However, the existence of CK10 together with co-expressed $\alpha$-actin and vimentin suggests that the corneas investigated were under oxidative stress and showed evidence of squamous metaplasia.
\end{abstract}

\section{Introduction}

A healthy ocular surface (both conjunctiva and cornea) is essential for clear and comfortable vision. Corneal epithelial maintenance has been attributed to a healthy population of putative limbal stem cells (LSC). Deficiency of limbal stem cells results in ocular surface disorders which lead to eye conjunctivitis, opacification and blindness, and provides challenging clinical problems. In order to treat severe ocular

Correspondence to: Professor James A. Ross, Tissue Injury and Repair Group, University of Edinburgh - MRC Centre for Regenerative Medicine, The Chancellor's Building, 49 Little France Crescent, Edinburgh EH16 4SB, Scotland, UK

E-mail: j.a.ross@ed.ac.uk

Key words: cornea, limbus, epithelium, stem cells, LGR5 disorders, it was hoped that therapy based on the limbal stem cell concept, such as ex vivo expanded limbal stem cells, would lead to transplantation strategies resulting in restoration of the ocular surface with stratified squamous epithelium and a clinically normal cornea. The current treatments involve replenishing the depleted LSC from a donor source such as limbal stem cells from the healthy eye of the patient (autograft) or from a donor (allograft). Currently, the idea of utilising ex vivo expanded limbal stem cells has gained popularity. However, there are limitations and concerns regarding the ex vivo culture of limbal stem cell approach that include the potential for infections, limited supply of donor tissue and the absence of a well-defined specific limbal stem cell marker. In addition, long-term preservation of the corneal rims decreases the viability of limbal stem cells thereby reducing the quality of the available biological material for transplant.

Several groups have reported the gene expression profile of fresh limbal and corneal epithelial cells which has significantly contributed to the understanding of cellular pathways and phenotype $(1,2)$. Surprisingly, the molecular profiling of long-term organ culture media-stored corneal epithelium has not been reported in the literature. In this study we evaluated the gene expression profile of cells isolated from corneal rims preserved for an extended time in the Corneal Transplant Service Eye Bank, Manchester. We were not able to include in our study a group any short-term or even non-organ culture media-stored epithelium due to Eye Bank standards and regulations. In order to evaluate our hypothesis that long-term preservation of the native tissue may decrease epithelial quality and health, we investigated the expression profile of different cytokeratin $(\mathrm{CK})$ species, which can provide characteristic features of limbal deficiency and epithelial keratinisation. We also examined the expression of genes which are global markers of adult stem cells including p63 $\alpha$ (a marker of epithelial stem cells, which supports stem cell proliferation and regulation in epithelial cells) and ABCG2 (a marker of basal limbal epithelial stem cells) but also LGR5, which has recently been identified as a novel stem cell marker (3). Strong p63 expression qualifies tissue for transplantation and predicts successful engraftment as it contains stem cells with remarkable p63-dependent proliferative capacity. Proliferating cells multiply and migrate 
to repair a corneal wound. However, this process can be irreversible and also lead to terminal differentiation of limbal stem cells (4). Profiling of different cytokeratin (CK3, CK12, CK10 and CK19) species in the cornea is therefore very important. Moreover, there is some disagreement among specialists that the colony-forming percentage is not the best marker for successful transplantation and that p63 expression should be considered as the most important marker. We propose that LGR5 deserves attention as a limbal stem cell molecular marker. The LGR5 gene encodes an orphan $\mathrm{G}$ protein-coupled receptor characterized by a large leucine-rich extracellular domain and seven transmembrane domains. Initially it was identified as a Wnt target gene expressed in colon cancer (5) but recently it has been identified as a novel stem cell marker of the intestinal epithelium and the hair follicle (3). However, in adult mice, it was reported that LGR5 expression is restricted to rare, scattered cells in intestine, eye, brain, stomach and reproductive organs (6). These findings might support the hypothesis that LGR5-positive cells may be associated with cellular stemness. Thus, LGR 5 may represent a general marker for adult stem cells but its molecular function is still unknown. This study provides a molecular profile of organ-culture-stored corneal epithelium and provides evidence that LGR5 is a molecular marker for rare limbal stem cells.

\section{Materials and methods}

Source of tissue. Surplus corneal buttons that were available through ethical permission for research purposes were used in these experiments. Human donor corneas from 5 donors, between 50 and 88 years of age, were obtained from the Manchester Eye Bank. All corneas were received from donors with specific consent for research purposes. All eyes were retrieved within $36 \mathrm{~h}$ of cadaver time and were stored for an extended time using the long-term organ culture technique according to standard CTS Eye Bank operating procedures (7). The obtained corneas had a mean time from death to enucleation of $29 \mathrm{~h}$ (range 12-23 h), a mean time from death to culture of $24 \mathrm{~h}$ (range 18-26 h) and a mean time in storage of 1 month (range 4-7 weeks).

Isolation and establishment of epithelial cells from whole corneas. The corneal rings were washed with CnT-20 medium, specifically formulated for human corneal epithelium and supplements included antibiotic/antimycotics (Cell N Tec, advanced cell systems). Tissue was cut diagonally into 4 identical pieces, which were incubated overnight in $5 \mathrm{ml}$ of $10 \mathrm{mg} / \mathrm{ml}$ dispase in CnT-20 medium at $4^{\circ} \mathrm{C}$. Next day each tissue section was carefully transferred into a small drop of CnT-20 (1-2 ml) and oriented with the epithelial cells upwards. Epithelium from whole corneas was scraped off gently and epithelial sheets were transferred on a small drop of TrypLe (Invitrogen) into a petri dish and incubated for $15 \mathrm{~min}$ at room temperature and dissociated into single cells by pipetting. After addition of a small amount of CnT-20 medium (1-2 ml) the mixture of cells in TrypLE and CnT-20 was centrifuged at $1000 \mathrm{rpm}$ for $5 \mathrm{~min}$. The freshly isolated limbal epithelial cells (LEC) were taken for further investigations. All isolated cells were sampled from the same surface area to retain significant similarities in the phenotype of the cells.
Immunocytochemistry. The expression of selected epithelial stem cell-related markers was confirmed by immunocy tochemistry using p63 $\alpha$, ABCG2 and LGR5. Immunocytochemical analysis was performed as previously described (8) using an avidin-biotin complex (ABC) method. The peroxidase was then developed using DAB to produce end-products. Briefly, cells were fixed in cold acetone for $10 \mathrm{~min}$ and then incubated overnight at $4^{\circ} \mathrm{C}$ with primary antibodies, which were a mouse monoclonal antibody for human p63 $\alpha$ (1:100; Santa Cruz Biotechnology, Inc.), a rabbit polyclonal antibody for human ABCG2 (1:50; Santa Cruz Biotechnology, Inc.), a rabbit polyclonal antibody for human LGR5 (1:50; Abcam). Secondary antibody was applied for $1 \mathrm{~h}$ at room temperature. The cells were finally counterstained with haematoxylin and then visualized and images captured using a Leica DM IRB microscope. Negative controls were employed to test the specificity of each antibody.

Total RNA isolation. Total RNA was extracted from each sample ( $n=5$ for isolated limbal epithelial cells) using an RNeasy kit (Qiagen) according to the manufacturer's protocol. RNA was quantified by NanoDrop ND-1000 (Labtech International) and the integrity was established by Bioanalyzer (Agilent Technologies) analysis.

Reverse transcription-polymerase chain reaction and quantitative real-time polymerase chain reaction. In order to analyse the gene expression profile, several genes were selected for reverse transcription-polymerase chain reaction (RT-PCR) analysis, using total RNA derived from freshly isolated epithelial cell samples. The quantitative validation of the expression of selected genes was performed by QRT-PCR on Custom TaqMan array plates (Applied Biosystems). Firstly, $1 \mu \mathrm{g}$ of RNA was reverse transcribed to cDNA using random hexamer primers, $25 \mathrm{mM} \mathrm{MgCl}_{2}$, 10X RT buffer, $10 \mathrm{mM}$ dNTPs, AMV (HC), RNAsin (all reagents from Promega, Madison, WI; http:// www.promega.com). The resulting mixture was incubated at $42^{\circ} \mathrm{C}$ for $60 \mathrm{~min}$ and $95^{\circ} \mathrm{C}$ for $5 \mathrm{~min}$. RT-PCR reactions $(\mathrm{n}=5$ for isolated limbal epithelial cells) were performed with master mix [Taq buffer, $25 \mathrm{mM} \mathrm{MgCl}$, $10 \mathrm{mM}$ dNTPs, Taq-polymerase (1:5), $10 \mu \mathrm{M}$ primers, $\mathrm{dH}_{2} \mathrm{O}$, cDNA $(20 \mathrm{ng} / \mu \mathrm{l})$ ] (all reagents from Promega). RT-PCR reactions were performed using the Veriti 96-well Thermal cycler (Applied Biosystems), at $94^{\circ} \mathrm{C}$ for $5 \mathrm{~min}$, followed by 35 cycles at $94^{\circ} \mathrm{C}$ for $1 \mathrm{~min}, 56^{\circ} \mathrm{C}$ for $1 \mathrm{~min}, 72^{\circ} \mathrm{C}$ for $1 \mathrm{~min}$. The PCR products were resolved and visually analysed on a $2 \%$ agarose gel in $1 \mathrm{X}$ TAE (triacetate EDTA) running buffer. PCR amplicon size was estimated using a 1000-bp DNA ladder (Promega). Primer sequences are provided in Table I.

Quantitative validation of gene expression levels was assessed by quantitative real-time polymerase chain reaction (Q-PCR) (Applied Biosystems StepOne Real-Time PCR Systems) for isolated limbal epithelial cells $n=3$, using custom TaqMan arrays plates, applying the Universal TaqMan PCR master mix (Applied Biosystems, Foster City, CA, USA), following the manufacturer's protocol. Amplification was performed for each cDNA $(20 \mathrm{ng} / \mu \mathrm{l})$ sample in triplicate and gene expression level was normalized to GAPDH. The data were quantified and normalized using the $\Delta \mathrm{Ct}$ method. Primers for TaqMan arrays were designed by Applied Biosystems. 
Table I. Human primer sequences used for reverse transcription-polymerase chain reaction (RT-PCR).

\begin{tabular}{lllr}
\hline Gene name & \multicolumn{1}{c}{ Forward primer } & \multicolumn{1}{c}{ Reverse primer } & Product size (bp) \\
\hline GAPDH & TGCACCACCAACTGCTTAGC & GGCATGGACTGTGGTCATGAG & 50 \\
Pax6 & AACAGACACAGCCCTCACAAACA & CGGGAACTTGAACTGGAACTGAC & 275 \\
p63 & ATGCAGTACCTTCCTCAGCACACA & TTCCTGAAGCAGGCTGAAAGGAGA & 104 \\
ABCG2 & GTGCACATGCTTGGTGGTTTGT & AGCTCGGTCTTAACCAAAGGCTCA & 159 \\
LGR5 & CTCTTCCTCAAACCGTCTGC & GATCGGAGGCTAAGCAACTG & 181 \\
CK3 & AAGAACATGGAGGACCTGGTGGAA & AGGCACTGTCCACATCCTTCTTCA & 115 \\
CK12 & AGGCCATGGATCTCTCCAACAACA & TTCCAACACTGGAAGCAGACATGC & 123 \\
CK10 & TTGGTGGAGGTAGCTTTCGTGGAA & AGAAGGCCACCATCTCCTCCAAAT & 130 \\
CK18 & GCATCCAGAACGAGAAGGAG & ACTTTGCCATCCACTATCCG & 1027 \\
CK19 & TTGTCCTGCAGATCGACAAC & TCTTCCAAGGCAGCTTTCAT & 514 \\
$\alpha$-actin & GCAATTTGACGACCTCTTCC & TGTGCTCAACCACGAATGA & 70 \\
VIM & GAGAACTTTGCCGTTGAAGC & CGTGATGCTGAGAAGTTTCG & 343 \\
\hline
\end{tabular}

Table II. The mean \pm standard deviation (SD) averaged from three different donors of results with mRNA levels [p $<0.001$ for all investigated markers by analysis of variance (ANOVA)].

\begin{tabular}{lcccccccc}
\hline $\mathrm{n}=3$ & Pax6 & $\mathrm{p} 63$ & $\mathrm{CK} 3$ & CK12 & CK4 & CK18 & CK19 & VIM \\
\hline$\Delta$ Ct Mean & 2.55 & 7.26 & 2.78 & 2.53 & 2.53 & 3.75 & 1.34 & 2.75 \\
$\Delta$ Ct SE & 0.80 & 3.06 & 1.93 & 1.94 & 2.69 & 0.39 & 0.26 & 1.31 \\
F test & $5.12 \times 10^{29}$ & $1.22 \times 10^{20}$ & $2.69 \times 10^{26}$ & $7.03 \times 10^{18}$ & $3.05 \times 10^{24}$ & $4.1 \times 10^{26}$ & $1.34 \times 10^{24}$ & $4.81 \times 10^{27}$ \\
(p-value) & $<0.001$ & $<0.001$ & $<0.001$ & $<0.001$ & $<0.001$ & $<0.001$ & $<0.001$ & $<0.001$ \\
\hline
\end{tabular}

Statistical analysis. Statistical significance of the results was assessed by the analysis of variance (ANOVA) and is reported in Table II. Analysis of variance in Table II showed differences in mRNA levels between three investigated samples for all molecular markers. This variation between samples could be explained by many factors including donor age, death-topreservation time and length of time in tissue storage.

\section{Results}

We used freshly isolated human corneal epithelial cells in order to reduce potential differentiation of isolated cells and the risk of contamination or disease transmission during culture. Immunocytochemical staining of freshly-isolated corneal limbal epithelial cells (Fig. 1) for the expression of the putative stem cells markers ABCG2, p63 $\alpha$ and LGR5 revealed positive staining for ABCG2 and p63 $\alpha$, indicating the existence of side-population cells with a stem cell phenotype. There was also very strong staining for LGR5, which has been suggested to be a marker for limbal stem cells in adult tissues (3). ABCG2 is expressed actively on the cellular surface, however cytoplasmic localisation is also possible (9). In order to confirm these observations gene expression was further investigated by PCR.

RNA prepared from isolated corneal epithelial cells, from the limbal area of the cornea, was of very high quality (260/280, 260/230 in the range of 1.89-2.0; RIN 8.0-9.0. RT-PCR (Fig. 2) demonstrated mRNA expression of ABCG2, p63 $\alpha$ and LGR5,
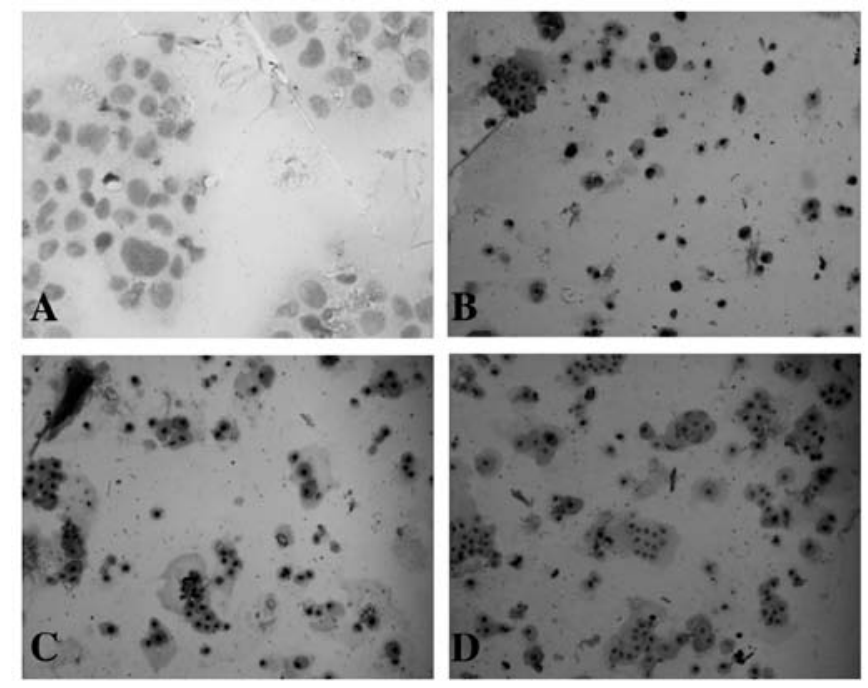

Figure 1. Immunocytochemistry images of adult stem cells markers in isolated limbal epithelial cells stained with LGR5, ABCG2 and p63. (A) Negative control, expression of LGR5 (B), ABCG2 (C) and p63 (D).

cytokeratin (CK), family members, vimentin and $\alpha$-actin, with GAPDH as an internal control. Analysis of the the mRNA encoding corneal differentiation markers showed expression of CK12 and CK3 (Figs. 2B and 3). In addition, quantitative validation of the expression level of selected genes was analysed using custom TaqMan array plates (Fig. 3). The expression 
A

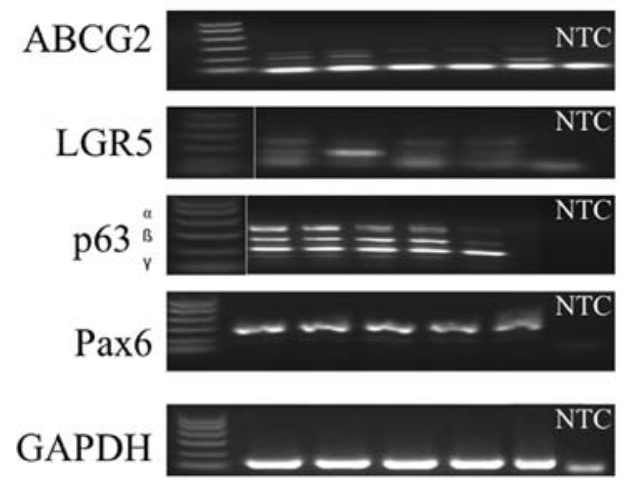

C
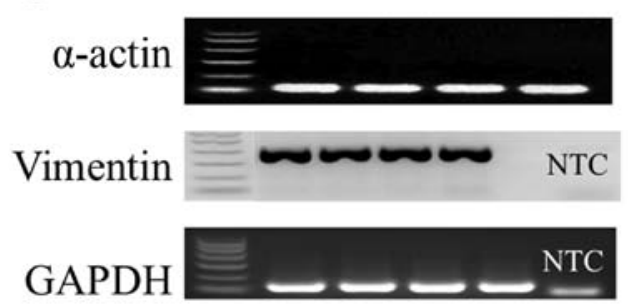

B

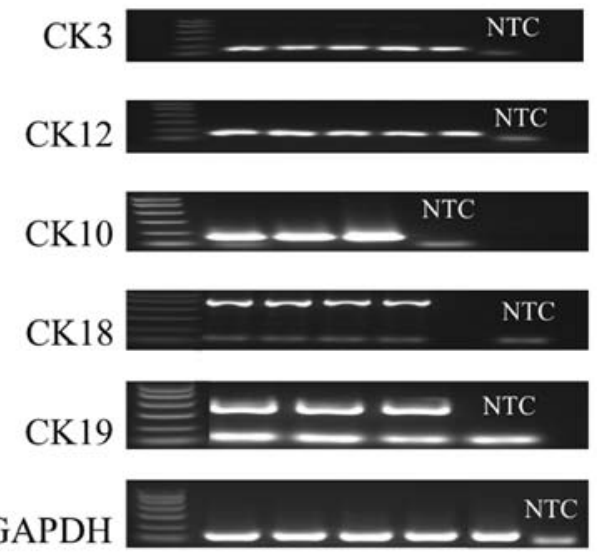

Figure 2. Reverse transcription polymerase chain reaction (RT-PCR) analysis of isolated limbal epithelial cells for selected molecular markers. (A) ABCG2, LGR5, p63, Pax6 expression in isolated epithelial cells (5 tissues). (B) Cytokeratins expression in isolated epithelial cells (3-5 tissues). (C) $\alpha$-actin and vimentin expression in isolated epithelial cells (4 tissues).

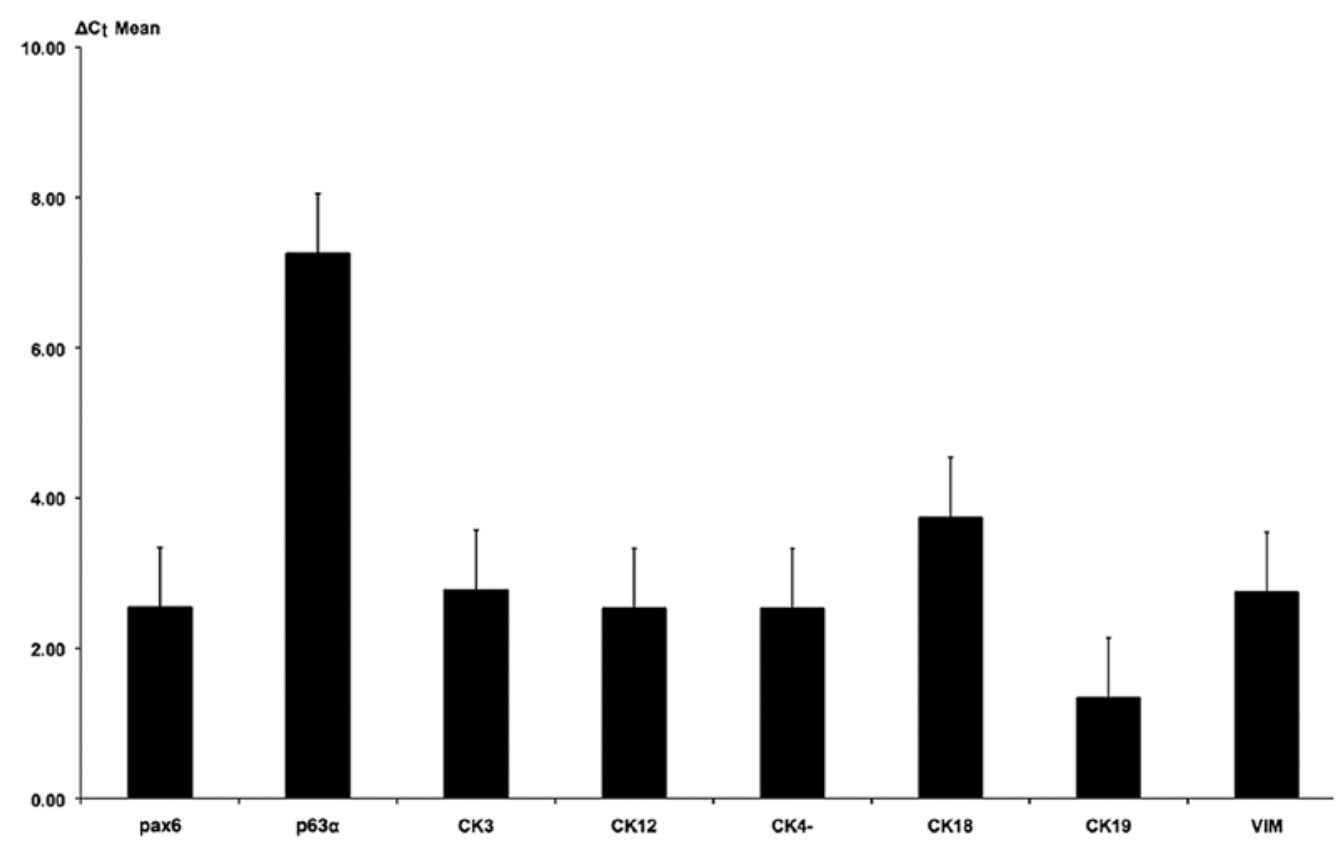

Figure 3. Quantitative real-time reverse transcriptase polymerase chain reaction (Q-PCR) analysis for expression of selected corneal epithelial markers. Data are an average of triplicates for three different donors. The gene expression level was normalized to GAPDH, a housekeeping gene used as an internal control with no difference in the mRNA level. Presented data were quantified and normalized using the $\Delta \mathrm{Ct}$ method.

level of p63 $\alpha$, Pax6, CK3, CK12, CK4, CK18, CK19 and vimentin mRNA were quantified with GAPDH as an internal control. The results of Q-PCR for nuclear transcription factor p63 $\alpha$ confirmed the findings of conventional RT-PCR and p63 $\alpha$ was expressed and quantified as being expressed at a low level. In addition, there was abundant expression of Pax6, the molecular marker responsible for eye morphogenesis, which regulates cell proliferation and determines differentiation.
Thus, weak expression of p63 $\alpha$ (Fig. 3) and a positive mRNA signal for ABCG2 and LGR5 (Fig. 2A) suggest that a small fraction of freshly isolated cells are derived from the limbal basal epithelium. However, strong complex expression patterns of other cytokeratins together with CK 3 and CK12 indicate the existence of the very specific corneal epithelial cell phenotype. Expression of both groups of markers suggests that there are cell cultures which are heterogeneous and contain both stem 
cells and differentiated epithelial cells. We have also detected signals from CK4 and CK18, both components of stratified squamous epithelium. There was also mRNA expression for CK10 and $\alpha$-actin (Fig. 2B) accompanied by significantly increased expression of CK19 and vimentin (Figs. 2B and 3), which may indicate that isolated cells represent a pathological phenotype and that the corneas investigated present features consistent with limbal stem cell deficiency.

\section{Discussion}

In this study we have evaluated the molecular state of the corneal limbal epithelial/stem cells isolated from whole corneas that have been in extended storage. All isolated cells have been sampled from the same surface area to retain significant similarities in cell phenotype.

Enucleated corneas were stored in organ culture medium (MEM 2\% FBS). The time interval from death of the donor to storage of the cornea is generally kept relatively short, within $12 \mathrm{~h}$ or less. In the case of our cadaveric donors this time exceeded $24 \mathrm{~h}$. Using the organ culture method, the storage period can be extended up to 4-5 weeks. Regarding our investigated material, the storage period was extended in some samples up to 7 weeks. We were not able to include in our study a group of short-term or even non-organ culture media-stored epithelium due to the Eye Bank standards and regulations. However, in order to detect tissue which does not tolerate storage, according to European Eye Bank Rules, inspection of the endothelium after storage is always mandatory. Nevertheless, the main aim of organ culture of corneal rims is to preserve a healthy corneal endothelium rather than maintaining epithelial health. Thus the aim of this study was to investigate the molecular profile of isolated corneal epithelial cells that have been in storage for an extended time. Our results show a shift in the molecular signature of the corneal epithelial/stem cells with storage.

In this study we analysed three groups of molecular markers: stem cell-related markers (LGR5, ABCG2 and p63), cytokeratins, (CK3, CK12, CK4, CK18 and CK10, CK19) and two biomarkers of transdifferentiation ( $\alpha$-actin and vimentin). The appearance of $\alpha$-actin and vimentin in epithelial cells marks a pathological state of metaplasia. Our immunocytochemistry study on limbal epithelial cells showed staining for ABCG2, p63 and LGR5, which confirms previously published data $(10,11)$ and confirms that they can be used as identifying markers for adult limbal stem cells. Our results show that a proportion of isolated human corneal epithelial cells possess limbal stem cell markers, indicating that these cells were still able to maintain the corneal limbal stem cell phenotype following cell isolation.

Limbal stem cell markers were also detected by analysis using RT-PCR and, additionally, some markers by Q-PCR. However, expression of ABCG2, LGR5 or p63 $\alpha$ in some of the analysed samples appeared to be weaker by Q-PCR analysis (Fig. 3). Moreover, quantitative validation of $\mathrm{p} 63 \alpha$ showed weak expression of this molecular marker. This disparity between samples may be explained by deleterious effects during eye bank tissue preservation and by the influence of many other uncontrolled factors including donor age, deathto-preservation time and length of time in tissue storage.
These factors can influence the expression of mRNA for the progenitor cell markers of interest while the actual proteins themselves may be relatively better retained. In addition, the numbers of expected progenitor cells will be lower with decreasing quality of the stored material and one study (9), for example, found that ABCG2 positive cells are only $0.20 \%$ of the total limbal epithelial cells.

Nevertheless, analysis of p63 expression by standard RT-PCR analysis showed that isolated cells expressed three different p63 isoforms $(\alpha, \beta$ and $\gamma$ ) indicating that among isolated cells there were also stem cells with proliferative potential and self-renewal properties. Expression of the p63 marker is the best indicator of the existence of holoclones, which is the best prediction for successful engraftment. However, RT-PCR analysis of one isoform of p63 (p63 $\alpha)$ showed expression at a very low level indicating that isolated cells were predominantly transit amplifying cells from the central cornea, which could be differentiated into terminally differentiated corneal epithelial cells. This was also confirmed by strong co-expression of $\mathrm{CK} 3$ and CK12 and also CK10, CK19.

The existence of a stem cell niche in isolated cornea was additionally confirmed by LGR5 expression. This study, for the first time, shows that human corneal epithelial cells express LGR5 at both the mRNA and protein level suggesting that this molecule can be considered as a new molecular marker of corneal epithelial cells. In the stem cell niche of the murine hair follicle, LGR5 was identified in actively cycling cells and it has been shown that LGR5-positive cells maintain all cell lineages throughout long periods of time and build new hair follicles (12). LGR5 is a potential candidate for a definitive marker of human corneal epithelial limbal stem cells and provides great promise for future cornea regenerative medicine. However, more studies are needed to fully define and characterize LGR5 expression in different corneal regions as well as to validate its function.

Additionally, we also profiled cytokeratin expression in corneal cells. One study (13) has described a correlation between stem cell-related markers (ABCG2 and p63) and differentiation markers (cytokeratins CK3 and CK12) in progenitor cells. Our results show that extended organcultured tissue retains a heterogeneous population of cells that include putative stem cells, normal differentiated cells and a proportion of epithelial cells expressing abnormal differentiation markers. The other cytokeratins that are major components of the corneal epithelium are CK4 and CK18. CK4 is a widespread component of stratified squamous epithelium, however, the existence of CK18 in adult human corneal epithelium is still a matter of controversy (14). Our results indicate that the isolated cells had high expression of both cytokeratins CK4 and CK18. This distribution pattern of cytokeratins, which belong to the intermediate-filament proteins, is very specific for corneal epithelium, is not encountered elsewhere and is characteristic of the terminal differentiation pathway of corneal epithelial cells. In this study, abundant expression of CK19 and vimentin was also detected (Fig. 3). Vimentin is an intermediate filament that is largely expressed in stromal cells and less differentiated cells. CK19 is predominantly a cytokeratin of conjunctival cells. Other researchers (15), have found vimentin positivity in LEC within the basal and suprabasal cells. Vimentin and CK19 
have been reported $(16,17)$ as being expressed by the same groups of cells. Thus the LEC represent a niche containing a heterogeneous population of predominantly epithelial cells Moreover, it has been suggested that the loss of limbal stem cells through pathological processes may result in migration of conjunctiva-derived cells to the corneal surface. Therefore, the presence of conjunctiva-derived cells (cytokeratin 19 positive cells) is interpreted as a feature of limbal stem cell deficiency and is confirmed by downregulation of progenitor stem cells markers (LGR5, ABCG2 and p63). Another study (18), also reported vimentin in a variety of limbal dysplastic lesions. However, vimentin expression has also been reported as a feature of corneal regeneration and been found in the epithelium of keratokonus specimens (19).

Our analysis of cornea-specific markers has also demonstrated abundant expression of Pax6 (Fig. 3). Pax6 is a key factor in controlling the phenotype of ocular surface epithelia (20) and Pax6 expression is downregulated in squamous metaplasia. Biochemically, squamous metaplasia is characterised by the loss of cornea-specific keratins, CK3 and CK12, and by upregulation of CK1 and CK10. In our study there was a very strong co-expression of Pax6 and CK12. However, PCR profiling of corneal molecular markers also showed expression of CK10 (Fig. 2B), which suggests that there is an indication of abnormal differentiation. The concept of abnormal differentiation is strengthened by the observation of $\alpha$-actin and vimentin in epithelial cells, which mark the pathological state of metaplasia (21). Gene expression of CK10, $\alpha$-actin and vimentin suggest that the epithelial cells in storage are undergoing some form of stress potentially induced by environmental factors during the period of corneal preservation. These findings support our hypothesis that long-term preservation may decrease epithelial quality and health. Nevertheless, abundant expression of Pax6, CK3 and CK12 suggest that isolated cells retain phenotypic integrity.

In this study we have shown that long-term organ culturepreserved corneal epithelial tissues have a heterogeneous population of cells that express genes consistent with stem cells (mRNA encoding p63, ABCG2 and LGR5 and immunostaining for these markers) and cells with a differentiated phenotype (abundant expression of cytokeratins 12 and CK3). A proportion of cells expressed genes (CK10, $\alpha$-actin and vimentin) that are not normally expressed by corneal or limbal epithelial cells. The expression of CK10, $\alpha$-actin and vimentin may mark the abnormal cell differentiation associated with metaplasia. This finding may be important in further development of stem cell culture techniques. Storage- and culture-induced cell changes may affect the clinical outcome and usefulness of donor tissues and these factors should be taken into consideration in future clinical translational studies.

\section{Acknowledgements}

Joanna Brzeszczynska would like to thank Daphne Jackson Trust (DJT) for financial support provided by The Leverhulme Trust.

\section{References}

1. Zhou M, Li XM and Lavker RM: Transcriptional profiling of enriched populations of stem cells versus transient amplifying cells. A comparison of limbal and corneal epithelial basal cells. J Biol Chem 281: 19600-19609, 2006.

2. Diehn JJ, Diehn M, Marmor MF and Brown PO: Differential gene expression in anatomical compartments of the human eye. Genome Biol 6: R74, 2005.

3. Haegebarth A and Clevers H: Wnt signaling, lgr5, and stem cells in the intestine and skin. Am J Pathol 174: 715-721, 2009.

4. BarbaroV, Testa A, Di Iorio E, Mavilio F, Pellegrini G and De Luca M: C/EBP regulates cell cycle and self-renewal of human limbal stem cells. J Cell Biol 177: 1037-1049, 2007.

5. Van der Flier LG, Sabates-Bellver J, Oving I, Haegebarth A, De Palo M, Anti M, van Gijn ME, Suijkerbuijk S, van de Wetering M, Marra G and Clevers H: The intestinal Wnt/TCF signature. Gastroenterology 132: 628-632, 2007.

6. Shackleton M, Vaillant F, Simpson KJ, Stingl J, Smyth GK, Asselin-Labat ML, Wu L, Lindeman GJ and Visvader JE: Generation of a functional mammary gland from a single stem cell. Nature 439: 84-88, 2006.

7. Armitage WJ and Easty DL: Factors influencing the suitability of organ-cultured corneas for transplantation. Invest Ophthalmol Vis Sci 38: 16-24, 1997.

8. Bentley D, Selfridge J, Millar JK, Samuel K, Hole N, Ansell JD and Melton DW: DNA ligase I is required for foetal liver erythropoiesis but is not essential for mammalian cell viability. Nat Genet 13: 489-491, 1996.

9. Watanabe K, Nishida K, Yamato M, Umemoto T, Sumide T, Yamamoto K, Maeda N, Watanabe H, Okano T and Tano Y: Human limbal epithelium contains side population cells expressing the ATP-binding cassette transporter ABCG2. FEBS Lett 565: 6-10, 2004.

10. Pellegrini G, Dellambra E, Golisano O, Martinelli E, Fantozzi I, Bondanza S, Ponzin D, McKeon F and De Luca M: p63 identifies keratinocyte stem cells. Proc Natl Acad Sci USA 98: 3156-3161, 2001.

11. Krulova M, Pokorna K, Lencova A, Fric J, Zajicova A, Filipec M, Forrester JV and Holan V: A rapid separation of two distinct populations of mouse corneal epithelial cells with limbal stem cell characteristics by centrifugation on percoll gradient. Invest Ophthalmol Vis Sci 49: 3903-3908, 2008.

12. Jaks V, Barker N, Kasper M, van Es JH, Snippert HJ, Clevers H and Toftgård R: Lgr5 marks cycling, yet long-lived, hair follicle stem cells. Nat Genet 40: 1291-1299, 2008.

13. De Paiva CS, Chen Z, Corrales RM, Pflugfelder SC and Li DQ: ABCG2 transporter identifies a population of clonogenic human limbal epithelial cells. Stem Cells 23: 63-73, 2005.

14. Kasper M, Stosiek P and Lane B: Cytokeratin and vimentin heterogeneity in human cornea. Acta Histochem 93: 371-381, 1992.

15. Shanmuganathan VA, Foster T, Kulkarni BB, Hopkinson A, Gray T, Powe DG, Lowe J and Dua HS: Morphological characteristics of the limbal epithelial crypt. Br J Ophthalmol 91: 514-519, 2007.

16. Joseph A, Powell-Richards AO, Shanmuganathan VA and Dua HS: Epithelial cell characteristics of cultured human limbal explants. Br J Ophthalmol 88: 393-398, 2004.

17. Lauweryns B, van den Oord JJ and Missotten L: The transitional zone between limbus and peripheral cornea. An immunohistochemical study. Invest Ophthalmol Vis Sci 34: 1991-1999, 1993.

18. Dushku N and Reid TW: P53 expression in altered limbal basal cells of pingueculae, pterygia, and limbal tumors. Curr Eye Res 16: 1179-1192, 1997.

19. SundarRaj N, Rizzo JD, Anderson SC and Gesiotto JP: Expression of vimentin by rabbit corneal epithelial cells during wound repair. Cell Tissue Res 267: 347-356, 1992.

20. Li W, Chen YT, Hayashida Y, Blanco G, Kheirkah A, He H, Chen SY, Liu CY and Tseng SC: Down-regulation of Pax6 is associated with abnormal differentiation of corneal epithelial cells in severe ocular surface diseases. J Pathol 214: 114-122, 2008.

21. Ou Y, Geng P, Liao GY, Zhou Z and Wu WT: Intracellular GSH and ROS levels may be related to galactose-mediated human lens epithelial cell apoptosis: role of recombinant hirudin variant III. Chem Biol Interact 179: 103-109, 2009. 